\title{
Análise de Objetivos Educacionais Utilizando a Taxonomia de Bloom Revisada Aplicada a um PPC do Curso de ADS
}

\author{
Mário W. Pereira ${ }^{1}$, Luiza S. Pontello ${ }^{2}$ \\ ${ }^{1}$ Instituto Federal de Educação, Ciência e Tecnologia do Ceará - Campi Horizonte - CE \\ - Brazil \\ ${ }^{2}$ Instituto Federal de Educação, Ciência e Tecnologia do Ceará - Campi Fortaleza - CE \\ - Brazil \\ mario.wilson@ifce.edu.br, pontellodifce.edu.br
}

\begin{abstract}
The focus of this research was to assess whether the educational objectives of the PPC of technological graduation in Systems Analysis and Development (ADS) of IFCE, Canindé campuses, are aligned with the professional profile of completion, corresponding to the National Catalog of Higher Technology Courses - MEC (CNCST), using the two-dimensional table of the Revised Bloom Taxonomy (TBR). Descriptive research was adopted as a methodology; as a data collection technique, documentary research; the analysis of the data being qualitative and quantitative. It was found that there is a misalignment, in the categories of the dimension of cognitive processes. It is recommended to review the objectives of the specific subjects of the course.
\end{abstract}

Resumo. O foco desta pesquisa foi avaliar se os objetivos educacionais do PPC da graduação tecnológica em Análise e Desenvolvimento de Sistemas (ADS) do IFCE, campi Canindé, estão alinhados com o perfil profissional de conclusão, correspondente ao Catálogo Nacional de Cursos Superiores de Tecnologia - MEC (CNCST), utilizando a tabela bidimensional da Taxonomia de Bloom Revisada (TBR). Adotou-se, como metodologia, a pesquisa descritiva; como técnica de coleta de dados, a pesquisa documental; sendo a análise dos dados qualitativa e quantitativa. Foi constatado que há um desalinhamento, nas categorias da dimensão dos processos cognitivos. Recomenda-se a revisão dos objetivos das disciplinas especificas do curso.

\section{Introdução}

O Projeto Pedagógico de Curso (PPC) é o instrumento que determina as diretrizes operacionais de um curso, nele, estão especificadas as motivações, as teorias, as metodologias, o currículo, o itinerário formativo e a avaliação que balizam a formação do estudante. Segundo o Instituto Federal de Educação, Ciência e Tecnologia do Ceará (2017b), em seu Manual para a Elaboração de Projetos Pedagógicos de Cursos Técnicos e de Graduação, o PPC deve estabelecer estratégias formativas coerentes com as áreas de conhecimento contempladas, sendo fundamental que se atente para seu processo de elaboração e atualização.

Os Programas de Unidade Didática (PUDs) do PPC estruturam o itinerário formativo a ser percorrido ao longo do processo de ensino e de aprendizagem, definindo os objetivos educacionais, propondo metodologias e formas de avaliação ao longo do curso, informações que amparam e auxiliam a prática docente, especialmente, dos 
professores que ingressam em novos campi e cursos. Por isso, é importante avaliar a qualidade e a conformidade dos objetivos educacionais, os quais, conforme Joye (2013), decorrem da análise das competências profissionais requeridas, definem as intenções perseguidas mediante um programa de formação, sendo a condição necessária à avaliação da aprendizagem. Pautando-se nos objetivos educacionais, é que se planeja, executa e avalia o processo de ensino e de aprendizagem.

É importante que a integração dessas metas promovam um currículo que assegure a formação do estudante segundo o perfil profissional de conclusão do curso (Zorzo, 2017). O PPC resultante deve ser compatível com as normativas legais institucionais e nacionais, destacando-se as diretrizes dos Catálogos Nacionais de Cursos Técnicos e Superiores. Para o Instituto Federal de Educação, Ciência e Tecnologia do Ceará (2017b), “a relação entre objetivos, perfil profissional e estrutura curricular é mérito de avaliação para aprovação de um PPC". Nessa temática, Ferraz e Belhot (2010) afirmam que decidir e definir os objetivos de aprendizagem significa estruturar, de forma consciente, o processo educacional para propiciar mudanças de pensamentos, ações e condutas, alinhados à formação das competências, de acordo com o perfil profissional delineado pela escola.

A elaboração e a verificação dos objetivos educacionais podem ser facilitadas pela utilização da taxonomia proposta por Bloom et al. (1956), a qual auxilia no planejamento, organização e controle das atividades didáticas em função daquilo que pretende que o aluno aprenda em nível de profundidade e complexidade. Sua utilização para a análise dos objetivos educacionais pode contribuir com os processos de planejamento e de reformulação dos PPCs para melhor qualificar a formação profissional pretendida e aperfeiçoar o processo de ensino e aprendizagem promovido pelos docentes, principalmente, os recém-ingressos no curso.

O projeto pedagógico do curso de Análise e Desenvolvimento de Sistemas (ADS) do Instituto Federal de Educação, Ciência e Tecnologia do Ceará (2017a), campi Canindé, foi elaborado no ano de 2017. A primeira turma de alunos do curso só iniciou dois anos depois, no semestre letivo de 2019.2. Nesse ínterim, todos os docentes da área específica, que participaram da construção do documento, foram removidos para outros campi da instituição, restando a outro grupo de professores colocar o curso em operação, realizar as avaliações e as atualizações pertinentes ao PPC. Para tal processo, este trabalho tem por finalidade avaliar se os objetivos educacionais do PPC da graduação tecnológica em ADS do IFCE Canindé estão alinhados com o perfil de conclusão, correspondente ao Catálogo Nacional de Cursos Superiores de Tecnologia (CNCST), utilizando a tabela bidimensional da Taxonomia de Bloom Revisada (TBR) como ferramenta de análise de dados.

\section{Fundamentação Teórica}

$\mathrm{Na}$ educação, decidir e definir os objetivos educacionais significa estruturar, de forma consciente, o processo educacional de modo a oportunizar mudanças de pensamentos, ações e condutas (FERRAZ; BELHOT, 2010). Mager (1979) afirma que o objetivo descreve um resultado que se pretende alcançar com o ensino, sendo uma base sólida para a seleção ou o planejamento de métodos, materiais ou conteúdos de aprendizagem. No contexto do IFCE, os objetivos educacionais estão inseridos no Programa de Unidade Didática (PUD), elaborado para cada componente curricular de um PPC. 
O uso de objetivos educacionais permite ao docente planejar, desenvolver e avaliar o processo de ensino e aprendizagem com base no que se espera do aluno e não apenas no conteúdo. Uma das teorias utilizadas para sistematizar esse trabalho é a Taxonomia de Bloom (BLOOM, 1979 apud SANTOS, 2016).

A Taxonomia de Bloom é um modelo amplamente empregado no contexto educacional. Ferraz e Belhot (2010) destacam que uma das vantagens dessa ferramenta é estimular os educadores a auxiliarem seus discentes, de forma estruturada e consistente, a adquirirem competências específicas de dominar habilidades mais simples (fatos) para, posteriormente, dominarem as mais complexas (conceitos).

A proposta dessa taxonomia consiste em três domínios: o cognitivo, o afetivo e o psicomotor, que caracterizam as habilidades, as capacidades e as atitudes que devem ser desenvolvidas no processo educacional (PIKARD, 2007 apud SANTOS, 2016). Conforme Ferraz e Belhot (2010), o domínio cognitivo envolve a aquisição de um novo conhecimento, do desenvolvimento intelectual, de habilidades e de atitudes; o domínio afetivo está associado à área emocional e afetiva, que incluem comportamentos, atitudes, responsabilidade, respeito, emoções e valores; o domínio psicomotor está relacionado a habilidades físicas próprias. Embora existam taxonomias específicas para objetivos do domínio cognitivo, do domínio afetivo e do domínio psicomotor, os três domínios são inter-relacionados e só artificialmente - e para finalidades didáticas - é que os distinguimos (MINISTÉRIO DA EDUCAÇÃO E CULTURA, 1979).

Ainda que todos os três domínios (cognitivo, afetivo e psicomotor) tenham sido amplamente discutidos e divulgados, em momentos diferentes e por pesquisadores diferentes, o domínio cognitivo é o mais conhecido e utilizado (FERRAZ; BELHOT, 2010). Neste trabalho, o foco da análise dos objetivos educacionais converge para o domínio cognitivo, considerando possíveis sobreposições com os domínios afetivo e psicomotor.

Após quatro décadas da criação da Taxonomia de Bloom, um coletivo de especialistas revisou e publicou, no ano de 2001, um livro intitulado: A Taxonomy for learning, teaching and assessing: a revision of Bloom's taxonomy for educational objectives (ANDERSON et al., 2001). A Taxonomia de Bloom Revisada (TBR) divide o domínio cognitivo em duas dimensões. De acordo com Silva e Martins (2014), a dimensão dos níveis de conhecimento identifica o saber a ser ensinado pelo professor, no qual os substantivos de um objetivo determinam o que ensinar, já a dimensão do processo cognitivo especifica a maneira utilizada para a aprendizagem desse saber, no qual o verbo do objetivo estabelece como ensinar.

A dimensão do conhecimento possui quatro categorias ou níveis de conhecimento: efetivo (conteúdo básico), conceitual (inter-relação dos conteúdos), procedural (como realizar alguma coisa) e metacognitivo (amplitude e profundidade de conhecimento), que são hierárquicos e cumulativos, ou seja, o mais simples é prérequisito para a próxima categoria mais complexa.

A dimensão dos processos cognitivos já existia na primeira versão da Taxonomia de Bloom e foi reestruturada, conforme Ferraz e Belhot (2010), continuando com seis categorias, que foram renomeadas como lembrar (reproduzir conteúdos), entender (conexão entre o novo e o conhecimento prévio), aplicar (executar um procedimento), analisar (compreender a inter-relação entre as partes), avaliar (julgar baseando-se em critérios) e criar (desenvolvimento de ideias novas e originais). $\mathrm{O}$ 
princípio da complexidade foi mantido, do simples para o complexo, atribuindo mais flexibilidade ao conceito cumulativo. Joye (2013) afirma que existem aprendizagens de baixo nível, relacionadas a conceitos, fatos e ideias, que consistem na repetição de um saber-fazer, já as aprendizagens de nível elevado requerem elementos de análise, síntese e criação.

A TBR relaciona os objetivos educacionais em uma tabela bidimensional, na qual a dimensão do conhecimento está disposta em quatro linhas e os processos cognitivos categorizados em seis colunas. Nas células, formadas pela intersecção das dimensões, são inseridos os objetivos (FERRAZ; BELHOT, 2010). Essa tabela auxilia no planejamento e na avaliação do processo de ensino e aprendizagem, podendo ser aplicada para estruturar planos de aula, unidades curriculares e projetos pedagógicos de cursos. Para cada objetivo educacional, o verbo de ação está associado a categoria da dimensão do processo cognitivo e os substantivos a categoria da dimensão do conhecimento. Uma representação da tabela bidimensional da Taxonomia de Bloom (TBR) é apresentada na Tabela 1.

Tabela 1 - Tabela bidimensional da Taxonomia de Bloom Revisada.

\begin{tabular}{|c|l|l|l|l|l|l|}
\hline \multirow{2}{*}{$\begin{array}{c}\text { Dimensão do } \\
\text { Conhecimento }\end{array}$} & \multicolumn{5}{|c|}{ Dimensão dos processos cognitivos } \\
\cline { 2 - 7 } & Lembrar & Entender & Aplicar & Analisar & Avaliar & Criar \\
\hline Efetivo & & & & & & \\
\hline Conceitual & & & & & & \\
\hline Procedural & & & & & & \\
\hline Metagognitivo & & & & & & \\
\hline
\end{tabular}

Fonte: Adaptado de ANDERSON et al., 2001.

Este trabalho se baseou no artigo de MARCHESAN (2018), que analisou a relação entre os objetivos educacionais de 21 componentes curriculares e o objetivo do PPC de um curso Técnico em Administração, de um Instituto Federal, os quais foram classificados quanto às dimensões do processo cognitivo e dos níveis de conhecimento, a partir da tabela bidimensional da TBR, e verificou-se coerência entre os objetivos na dimensão do conhecimento, com ênfase nas categorias procedural e conceitual. Na dimensão dos processos cognitivos, evidenciou-se uma diferença na ênfase das categorias dos planos de ensino (Entender, Aplicar) em relação aos objetivos do projeto pedagógico (Aplicar, Entender).

Outro trabalho similar foi elaborado por Neto (2012), que identificou e avaliou a tendência das habilidades cognitivas requeridas pelo acadêmico do curso de graduação em Ciências Contábeis da UNESC, utilizando a Taxonomia de Bloom, verificou-se que a maior incidência dos objetivos se enquadra nas categorias que exigem habilidades cognitivas menos complexas, que o PPC propõe objetivos mais complexos e que há equivalência entre as habilidades descritas nos planos de ensino com as exigidas dos acadêmicos na resolução das questões das provas. 


\section{Metodologia}

Em vista do objetivo do presente estudo, adotou-se como metodologia a pesquisa descritiva, que, para Gil (2002), tem como objetivo primordial a descrição das características de determinada população ou fenômeno, utiliza técnicas padronizadas de coletas de dados e proporciona novas visões sobre uma realidade já conhecida.

Utilizou-se, como técnica de coleta de dados, a pesquisa documental, que "valese de materiais que não receberam ainda um tratamento analítico, ou que ainda podem ser reelaborados de acordo com os objetivos da pesquisa" (GIL, 2002). As fontes documentais foram o CNCST e o PPC do curso de ADS do IFCE, campi Canindé, dos quais foram analisadas as metas do perfil profissional de conclusão (no caso do primeiro documento) e os objetivos educacionais dos PUDs das disciplinas regulares do curso (referente ao segundo documento). Quanto à abordagem, a análise dos dados foi qualitativa ao dividir os objetivos educacionais analisados nos componentes curriculares do PPC de ADS em dois grupos e quantitativa ao levar em consideração a quantidade de objetivos classificados na tabela bidimensional da TBR para cada categoria das duas dimensões do domínio cognitivo, os processos cognitivos e o conhecimento.

Ao iniciar a análise no CNCST, notou-se que os objetivos apresentavam caráter estritamente técnico, por isso, optou-se por montar duas tabelas bidimensionais para a análise do segundo documento, o PPC do curso de ADS. A primeira tabela foi composta por todos os objetivos educacionais dos componentes curriculares regulares do curso, denominada "todas as disciplinas regulares" e a segunda tabela foi estabelecida com as metas relacionadas somente às disciplinas específicas do curso, nomeada como "somente as disciplinas específicas". Ao todo foram construídas três tabelas bidimensionais da TBR: a primeira "perfil profissional de conclusão", com 14 objetivos classificados; a segunda "todas as disciplinas regulares", com 94 objetivos classificados de 29 PUDs analisados e a terceira "somente as disciplinas específicas", com 61 objetivos classificados de 20 componentes curriculares analisados. O fluxograma, ilustrado na Figura 2, representa o percurso metodológico da pesquisa.

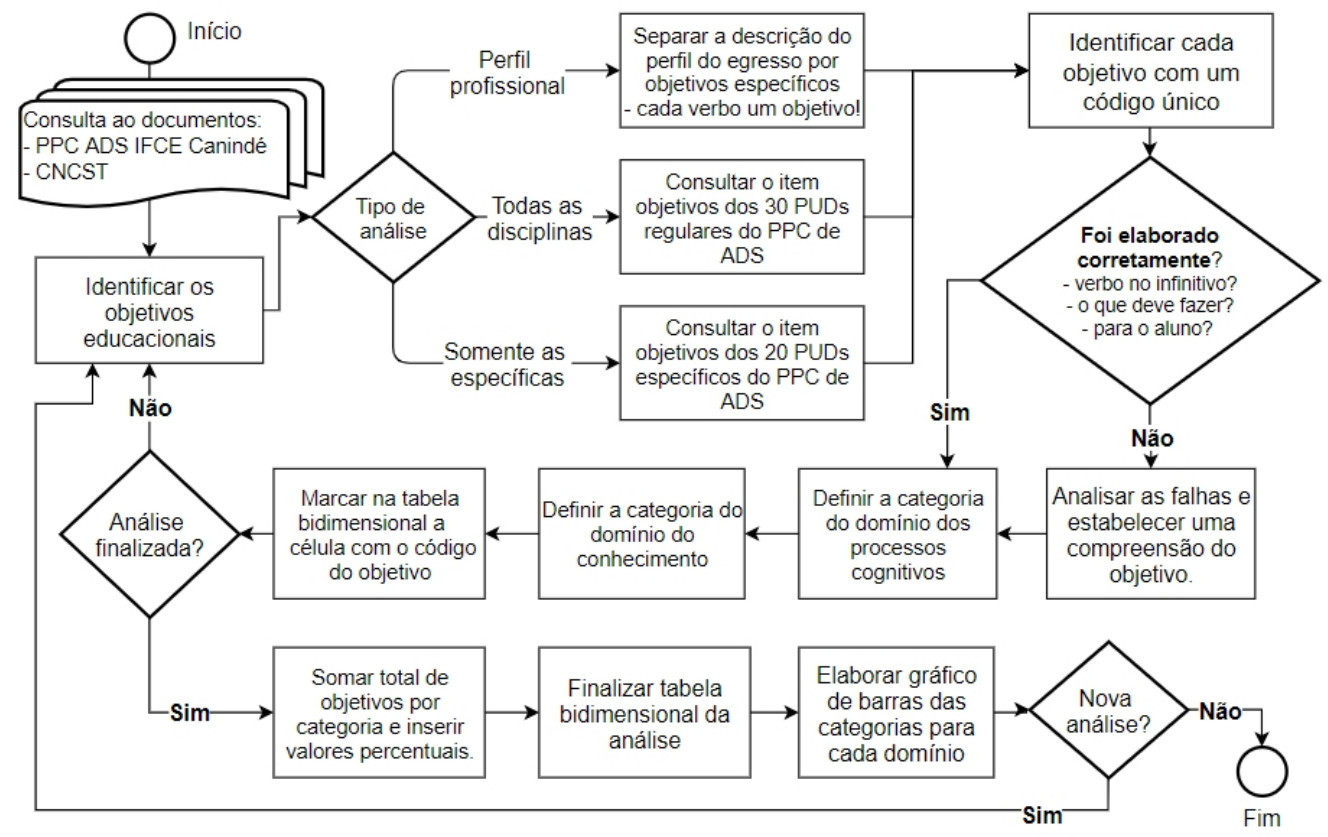

Figura 2 - Fluxograma do percurso metodológico da pesquisa. Elaborado pelo autor. 
As etapas do processo foram: consultar cada fonte documental para identificar os objetivos educacionais, em seguida, separar cada verbo de ação, atribuindo um código para sua identificação, depois, verificar se os objetivos estavam elaborados corretamente, caso contrário, interpretá-los e/ou corrigi-los a fim de estabelecer uma compreensão adequada. Após essa fase de refinamento, cada objetivo foi classificado nas categorias da dimensão dos processos cognitivos e do conhecimento e seu código foi inserido na célula correspondente da tabela bidimensional da TBR. Esse ciclo foi realizado até se esgotarem os objetivos de cada análise proposta. Com todos os códigos mapeados, os dados das células foram alterados para indicar a quantidade de objetivos mapeados em vez dos códigos, posteriormente, optou-se por adicionar novas linhas e colunas à tabela para contabilizar o total de objetivos educacionais por categoria e os respectivos valores percentuais. Por fim, para cada tabela elaborada, foram construídos dois gráficos de barras, um para as categorias da dimensão dos processos cognitivos e outro para as categorias da dimensão do conhecimento.

\section{Resultados e Discussões}

Em vista dos objetivos da pesquisa, os resultados estão apresentados no formato da tabela bidimensional da TBR, que foi adaptada com linhas e colunas para exibir os quantitativos e percentagens de cada categoria, além de um comparativo, com gráficos em barra, das dimensões dos processos cognitivos e do conhecimento de cada análise realizada. A sequência de apresentação das tabelas bidimensionais da TBR inicia com o perfil profissional de conclusão de ADS do CNCST, em seguida, todas as disciplinas regulares, por fim, somente as disciplinas específicas do curso.

O resultado da análise do perfil profissional de conclusão, conforme o CNCST, é apresentado na Tabela 2. Foram identificados quinze verbos de ação, que originaram o quantitativo de objetivos educacionais tabelados. Analisando-se a dimensão dos processos cognitivos, observou-se uma concentração nas categorias Aplicar $(21,43 \%)$, Analisar (28,57\%) e Avaliar (42,86\%), restando um objetivo na categoria Criar (7,14\%). Quanto à dimensão dos níveis de conhecimento, percebeu-se que a maior parte está classificada na categoria Procedural $(92,86 \%)$, um objetivo foi classificado como Metacognitivo (7,14\%). Como o catálogo foca no perfil profissional de conclusão do curso, a ausência de objetivos educacionais basilares resultou em algumas categorias iniciais com valor $0 \%$.

Tabela 2 - Classificação para o perfil profissional de conclusão, conforme o CNCST.

\begin{tabular}{|c|c|c|c|c|c|c|c|c|}
\hline \multirow{2}{*}{$\begin{array}{l}\text { Dimensão do } \\
\text { Conhecimento }\end{array}$} & \multicolumn{6}{|c|}{ Dimensão Processos Cognitivos } & \multirow{2}{*}{$\begin{array}{c}\text { Total } \\
\text { categoria }\end{array}$} & \multirow{2}{*}{$\begin{array}{l}(\%) \\
\text { categoria }\end{array}$} \\
\hline & Lembrar & Entender & Aplicar & Analisar & Avaliar & Criar & & \\
\hline Efetivo & 0 & 0 & 0 & 0 & 0 & 0 & $\mathbf{0}$ & $0 \%$ \\
\hline Conceitual & 0 & 0 & 0 & 0 & 0 & 0 & $\mathbf{0}$ & $0 \%$ \\
\hline Procedural & 0 & 0 & 3 & 4 & 5 & 1 & 13 & $92,86 \%$ \\
\hline Metacognitivo & 0 & 0 & 0 & 0 & 1 & 0 & 1 & $7,14 \%$ \\
\hline Total categoria & $\mathbf{0}$ & $\mathbf{0}$ & 3 & 4 & 6 & 1 & 14 & \\
\hline (\%) categoria & $0 \%$ & $\mathbf{0 \%}$ & $21,43 \%$ & $28,57 \%$ & $42,86 \%$ & $7,45 \%$ & & \\
\hline
\end{tabular}


O resultado da análise dos objetivos de aprendizagem dos vinte e nove componentes curriculares regulares do PPC de ADS é apresentado na Tabela 3. Identificou-se que, na dimensão dos processos cognitivos, há uma concentração nas categorias Lembrar $(23,40 \%)$, Entender $(24,47 \%)$ e Aplicar $(31,91 \%)$. As categorias mais elaboradas apresentaram baixo percentual: Analisar (6,38\%), Avaliar (6,38\%) e Criar (7,45\%). Quanto à dimensão do conhecimento, a ordem decrescente de percentual de objetivos classificados por categoria foi Procedural (45,74\%), Metacognitivo $(37,23 \%)$, Efetivo (10,64\%), Conceitual (6,38\%).

Tabela 3 - Classificação para todas as disciplinas regulares do PPC de ADS.

\begin{tabular}{|c|c|c|c|c|c|c|c|c|}
\hline \multirow{2}{*}{$\begin{array}{c}\text { Dimensão do } \\
\text { Conhecimento }\end{array}$} & \multicolumn{6}{|c|}{ Processos Cognitivos } & \multirow{2}{*}{$\begin{array}{c}\text { Total } \\
\text { categoria }\end{array}$} & \multirow{2}{*}{$\begin{array}{c}(\%) \\
\text { categoria }\end{array}$} \\
\hline & Lembrar & Entender & Aplicar & Analisar & Avaliar & Criar & & \\
\hline Efetivo & 8 & 2 & 0 & 0 & 0 & 0 & 10 & $10,64 \%$ \\
\hline Conceitual & 1 & 4 & 0 & 1 & 0 & 0 & 6 & $6,38 \%$ \\
\hline Procedural & 8 & 10 & 17 & 3 & 2 & 3 & 43 & $45,74 \%$ \\
\hline Metacognitivo & 5 & 7 & 13 & 2 & 4 & 4 & 35 & $37,23 \%$ \\
\hline Total categoria & 22 & 23 & 30 & 6 & 6 & 7 & 94 & \\
\hline$(\%)$ categoria & $23,40 \%$ & $24,47 \%$ & $31,91 \%$ & $6,38 \%$ & $6,38 \%$ & $7,45 \%$ & & \\
\hline
\end{tabular}

O resultado da análise dos objetivos de aprendizagem dos 20 componentes curriculares regulares e específicas do PPC de ADS é apresentado na Tabela 4. Dos 61 objetivos educacionais, constatou-se que, na dimensão dos processos cognitivos, há uma concentração nas categorias Lembrar (24,59\%), Entender (26,23\%) e Aplicar (31,15\%). Os grupos mais elevados apresentaram baixo percentual: Analisar $(8,20 \%)$, Avaliar $(3,28 \%)$ e Criar (6,56\%). Quanto à dimensão do conhecimento, a ordem decrescente de percentual de objetivos classificados por categoria foi Procedural (63,93\%), Metacognitivo (18,03\%), Efetivo (9,84\%), Conceitual (8,20\%).

Tabela 4 - Classificação dos objetivos para as disciplinas específicas do PPC de ADS.

\begin{tabular}{|c|c|c|c|c|c|c|c|c|}
\hline \multirow{2}{*}{$\begin{array}{l}\text { Dimensão do } \\
\text { Conhecimento }\end{array}$} & \multicolumn{6}{|c|}{ Processos Cognitivos } & \multirow{2}{*}{$\begin{array}{c}\text { Total } \\
\text { categoria }\end{array}$} & \multirow{2}{*}{$\begin{array}{c}(\%) \\
\text { categoria }\end{array}$} \\
\hline & Lembrar & Entender & Aplicar & Analisar & Avaliar & Criar & & \\
\hline Efetivo & 8 & 2 & 0 & 0 & 0 & 0 & 6 & $9,84 \%$ \\
\hline Conceitual & 1 & 4 & 0 & 1 & 0 & 0 & 5 & $8,20 \%$ \\
\hline Procedural & 8 & 10 & 17 & 3 & 2 & 3 & 39 & $63,93 \%$ \\
\hline Metacognitivo & 5 & 7 & 13 & 2 & 4 & 4 & 11 & $18,03 \%$ \\
\hline Total categoria & 15 & 16 & 19 & 5 & 2 & 4 & 61 & \\
\hline (\%) categoria & $24,59 \%$ & $26,23 \%$ & $31,15 \%$ & $8,20 \%$ & $3,28 \%$ & $6,56 \%$ & & \\
\hline
\end{tabular}


Para comparar a distribuição quantitativa dos objetivos educacionais, ao longo das categorias de cada dimensão, foram elaborados gráficos em barra, a partir das tabelas bidimensionais da TBR apresentadas, conforme a Figura 2.

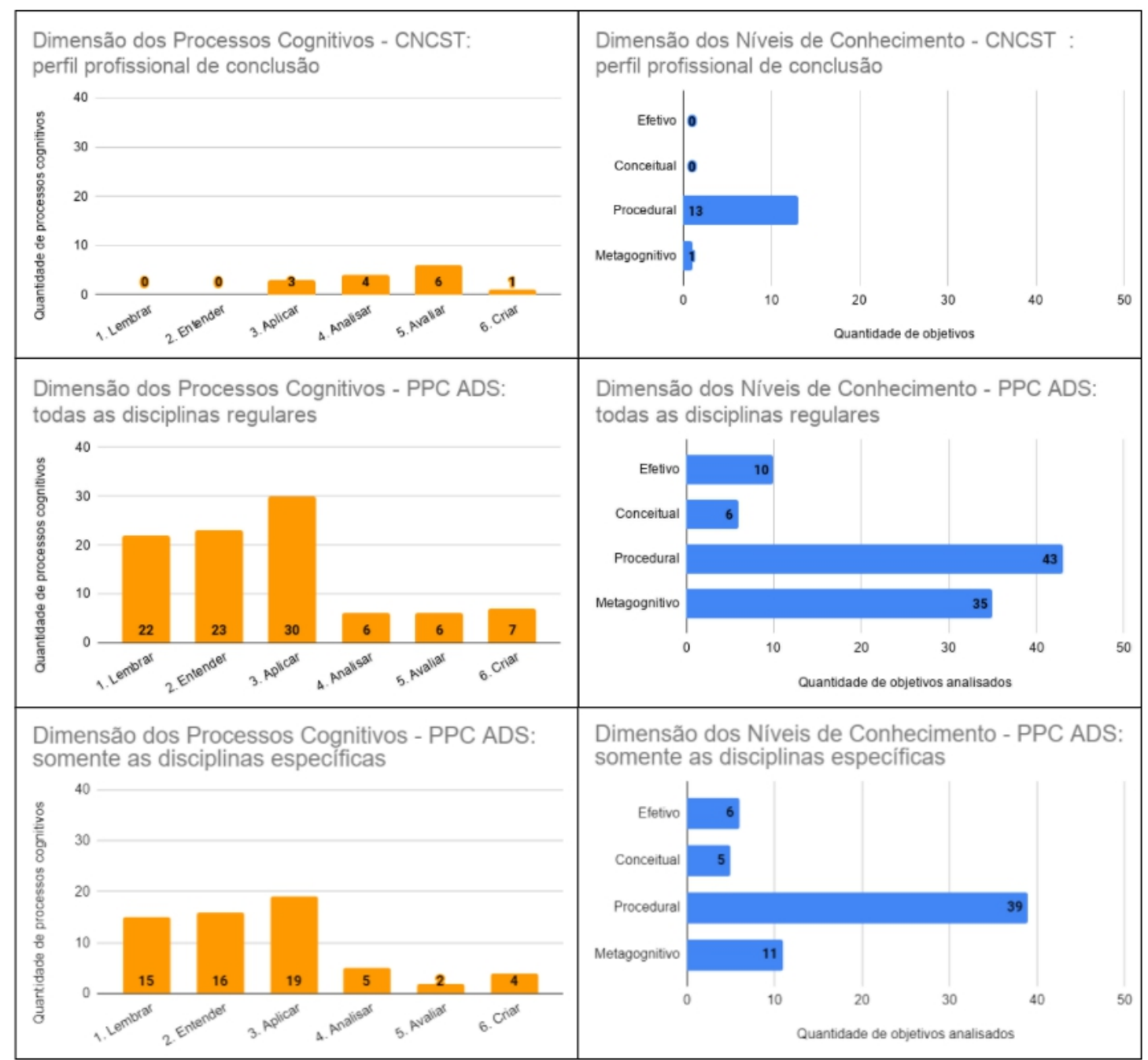

Figura 2 - Gráficos das tabelas bidimensionais da TBR analisadas.

Quanto à dimensão do processos cognitivos, os objetivos educacionais presentes no CNCST estão concentrados em categorias cognitivas mais elaboradas que o PPC do curso de ADS. Essa diferença pode ser explicada por Zorzo (2017), que afirma existirem algumas confusões no entendimento dos cursos superiores de tecnologia em relação aos cursos técnicos, que além da diferença no nível de formação, a capacitação dos profissionais de tecnologia exige o desenvolvimento de competências mais complexas do que as de nível técnico, com conhecimento tecnológico mais aprofundado, objetivos mais abrangentes e maior profundidade dos conteúdos programáticos.

Em relação à dimensão do conhecimento, todos os gráficos das tabelas analisadas apresentaram predominância de objetivos classificados na categoria Procedural, definidas por Ferraz e Belhot (2010) como "o conhecimento de como realizar alguma coisa utilizando métodos, critérios, algoritmos e técnicas"; características compatíveis com as competências e habilidades do egresso de ADS. 


\section{Conclusão}

Com o auxílio da tabela bidimensional da Taxonomia de Bloom Revisada, realizou-se a análise dos objetivos educacionais do perfil profissional de conclusão de ADS, conforme o Catálogo Nacional dos Cursos Superiores de Tecnologia, e dos 29 componentes curriculares regulares da graduação tecnológica em Análise e Desenvolvimento de Sistemas do IFCE, campi Canindé. Comparando-se as tabelas bidimensionais, avaliou-se que há um desalinhamento parcial entre o perfil profissional de conclusão de ADS no CNCST e o PPC do curso de ADS do IFCE Canindé. Na dimensão dos processos cognitivos da TBR, o primeiro documento apresentou concentração dos objetivos nas categorias superiores (Avaliar) e o segundo apontou convergência nas camadas inferiores (Aplicar). A falta de instrumentais, que auxiliem as comissões de elaboração e de revisão dos PPCs para definição e ajuste dos objetivos educacionais gerais e específicos, pode justificar esse desalinhamento entre caminho e destino, entre PPC e perfil profissional de conclusão. Recomenda-se a revisão da redação dos objetivos, principalmente, das disciplinas específicas do curso, a fím de ajustá-las às categorias Analisar e Avaliar da dimensão do processo cognitivo.

Esta pesquisa contribuiu, principalmente, com a análise dos objetivos educacionais dos componentes curriculares de um PPC, utilizando a tabela bidimensional da Taxonomia de Bloom Revisada, como forma de avaliar o alinhamento com o perfil profissional de conclusão de um curso do CNCST, em relação às categorias das dimensões (processos cognitivos e conhecimento) do domínio cognitivo da TBR. Ainda, auxilia na revisão da redação dos objetivos educacionais do PPC de ADS, indicando as falhas de elaboração. Futuramente, pretende-se realizar a comparação entre as tabelas bidimensionais da TBR do referido PPC de ADS do IFCE, campi Canindé, e do projeto pedagógico de um curso Técnico em Desenvolvimento de Sistemas, a fim de avaliar a similaridade das categorias das dimensões do domínio cognitivo entre o curso de nível superior e o seu correspondente em nível médio.

\section{References}

ANDERSON, L. W. et al. A taxonomy for learning, teaching, and assessing: A revision of Bloom's taxonomy of educational objectives. [S.1.]: Allyn \&Bacon, 2001.

BLOOM, B. S. Taxonomy of educational objectives, Handbook 1: Cognitive domain. Addison-Wesley Longman Ltd. New York: David McKay. 1956.

FERRAZ A. P. C. M.; BELHOT, R.V. Taxonomia de Bloom: revisão teórica e apresentação das adequações do instrumento para definição de objetivos instrucionais. Gest. Prod. 17(2):421-31; 2010.

INSTITUTO FEDERAL DE EDUCAÇÃO, CIÊNCIA E TECNOLOGIA DO CEARÁ. PPC - Tecnologia em Análise e Desenvolvimento de Sistemas. 2017a. Disponível em: $<$ https://ifce.edu.br/caninde/cursos/superiores/tecnologicos/ads/pdf/ppc-tecnologiaem-analise-e-desenvolvimento-de-sistemas.pdf/@@download/file/PPC\%20$\% 20$ Tecnologia $\% 20 \mathrm{em} \% 20 \mathrm{An} \% \mathrm{C} 3 \%$ A 1 lise $\% 20 \mathrm{e} \% 20$ Desenvolvimento $\% 20 \mathrm{de} \% 20 \mathrm{~S}$ istemas\%20.pdf> Acesso em: 14 out. 2020.

INSTITUTO FEDERAL DE EDUCAÇÃO, CIÊNCIA E TECNOLOGIA DO CEARÁ. Projeto Político-Pedagógico Institucional. 2018. Disponível em: $<$ https://ifce.edu.br/PPI.pdf $>$ Acesso em: 14 out. 2020. 
INSTITUTO FEDERAL DE EDUCAÇÃO, CIÊNCIA E TECNOLOGIA DO CEARÁ. Conselho Superior. Resolução Nº99, de 27 de setembro de 2017. Aprova o Manual para Elaboração de Projetos Pedagógicos de Cursos do IFCE. 2017b. Disponível em: $<$ https://ifce.edu.br/instituto/documentos-institucionais/resolucoes/2017/099-17aprova-o-manual-de-elaboracao-de-projetos-pedagogicos-de-cursos-doifce.pdf/@@download/file/099-17\%20\%20Aprova\%20o\%20Manual\%20de\%20Elabora\%C3\%A7\%C3\%A3o\%20de\%20Pr ojetos $\% 20$ Pedag\% $\%$. Acesso em: 13 out. 2020.

GIL, A. C. Como elaborar projetos de pesquisa. 4. ed. Atlas. São Paulo. 2002.

JOYE, C. R. Didáticas e Metodologias do Ensino Médio e da Educação Profissional. Instituto Federal de Educação, Ciência e Tecnologia do Ceará. SETEC-MEC. 137 p. 2013.

MAGER, R. F. A formulação de objetivos de ensino. Traduzido da segunda edição norte-americana por Cosete Ramos coma colaboração de Débora Karam Galarza. Proto Alegre, Globo. 1979.

MARCHESAN, M. R.; KUHN, M. C. Análise dos Objetivos Educacionais de um Curso Técnico em Administração pela Taxonomia de Bloom Revisada. Cadernos de Educação, n. 59, pp. 108-133. 2018. Faculdade de Educação UFPel. 2018. ISSN: 2178-079X.JOYE, C. R. Didáticas e Metodologias do Ensino Médio e da Educação Profissional. Instituto Federal de Educação, Ciência e Tecnologia do Ceará. SETECMEC. 137 p. 2013.

MINISTÉRIO DA EDUCAÇÃO. Catálogo Nacional de Cursos Superiores de Tecnologia. $\quad 3^{\circ}$ Edição. Brasília. 2016. Disponível em: $<$ http://portal.mec.gov.br/index.php?option $=$ com_docman\&view $=$ download\&alias $=9$ 8211-cncst-2016-a\&category_slug=outubro-2018-pdf-1\&Itemid=30192>. Acesso em: 13 out. 2020 .

MINISTÉRIO DA EDUCAÇÃO E CULTURA. Planejamento Curricular - decisões básicas do planejamento curricular. Documento III. SECRETARIA DE ENSINO $1^{\circ}$ E $2^{\circ}$ GRAUS. Brasília. 1979.

NETO, R. J.; DOS SANTOS, N.; DE ASSIS, B. C. Análise Das Habilidades Cognitivas Fundamentados Na Taxionomia De Bloom: Uma Análise No Curso De Ciências Contábeis. Seminário de Ciências Sociais Aplicadas, v. 3, n. 3, 2012.

SANTOS, R. S. F. D. Inserindo a Taxonomia Revisada de Bloom em um MOOC. Dissertação. Programa de Pós-Graduação em Ciência da Computação. Associação ampla entre a Universidade do Estado do Rio Grande do Norte e a Universidade Federal Rural do Semi-Árido. 2016.

SILVA, V. A. D.; MARTINS, M. I. Análise de questões de física do ENEM pela Taxonomia de Bloom Revisada. Revista Ensaio, Belo Horizonte, v. 16, n. 3, pp. 189202, 2014. Disponível em : <https://www.scielo.br/pdf/epec/v16n3/1983-2117-epec16-03-00189.pdf $>$. Acesso em: 13 out. 2020.

Zorzo, A. F. et al. Referenciais de Formação para os Cursos de Graduação em Computação. Sociedade Brasileira de Computação (SBC). 153p, 2017. ISBN 97885-7669-424-3. 